\title{
Development on depth of engagement intelligent measurement device for handcart circuit breaker contacts about metal-enclosed armored high voltage switchgear
}

\author{
Zhihui $\mathrm{Lu}^{1,2}$, Fuyun Zhu ${ }^{1,2}$, Hongjun $\mathrm{Ni}^{1}$, Shuaishuai $\mathrm{Lv}^{1}$ and Xingxing Wang ${ }^{1, \mathrm{a},{ }^{*}}$ \\ ${ }^{1}$ School of Mechanical Engineering, Nantong University, Nantong, Jiangsu, 226019, China \\ ${ }^{2}$ Nantong Power Supply Company, Jiangsu Electric Power Company, Nantong, Jiangsu, 226000, China \\ àwangxx@ntu.edu.cn (* corresponding author)
}

Keywords: HV switchgear, circuit breakers, contacts, depth of engagement, measurement devices.

\begin{abstract}
In order to further improve the measurement accuracy and convenience, a novel depth of engagement intelligent measurement device for handcart circuit breaker contacts about metal-enclosed armored high voltage switchgear was developed. The device includes six sets of precision displacement measurement sensors and mainframe box. Six sets of displacement sensors were installed in the six movable contacts internal counter bore of $\mathrm{A}, \mathrm{B}, \mathrm{C}$ three-phase of high voltage switchgear handcart circuit breaker, the power lines and signal lines were lead by a peripheral ring gap plum contacts and circuit breaker handcart. With the main data processing interface box connected to the main chassis after the data processing unit, the output is displayed on mainframe box of the data display. The device structure is reasonable, easy to measure and accurate. Its measurement range is $0-50 \mathrm{~mm}$; its measurement accuracy is $\pm 0.1 \mathrm{~mm}$.
\end{abstract}

\section{Introduction}

With the development of social economy and the power industry, intelligent substation has become one of the hot issues of the smart grid [1-4]. High voltage switchgear is important to power system electrical equipment. In addition, it's the key equipment of intelligent substation. High voltage switchgear has the functions of closing and disconnecting the power lines, as well as, protecting the security of power system. The reliability of closing and disconnecting the power lines of metal-enclosed armored high voltage switchgear equipment (hereinafter referred to as switchgear) depends on the engagement circumstances of handcart circuit breaker. Now power system is unattended basically because of the widely used on-line monitoring technology [5-9]. But the installation and commissioning method about switchgear circuit breaker handcart still remains in the traditional stage during the substation construction period. The traditionally method is smearing a layer of Vaseline on the static contact, and pushing the trolley to the operating position, then pulling the handcart outside the cabinet, measuring residual indentation of Vaseline when static and dynamic contact handcart remain on the stationary contact. The dimensional data error of this original measurement method is large, especially in hot summer, the Vaseline liquefies at the above $35{ }^{\circ} \mathrm{C}$, the indentation is not obvious, which causes great difficulties to site measurements and acceptance. Further, since the switch mechanism will be deformed after long-term use, the push position of each switch is not completely precise and consistent.

Therefore, in order to further improve the measurement accuracy and convenience, this paper developed a depth of engagement intelligent measurement device for handcart circuit breaker contacts about metal-enclosed armored high voltage switchgear.

\section{Scheme design}

Figure 1 shows the schematic diagram on depth of engagement intelligent measurement device for switchgear. 


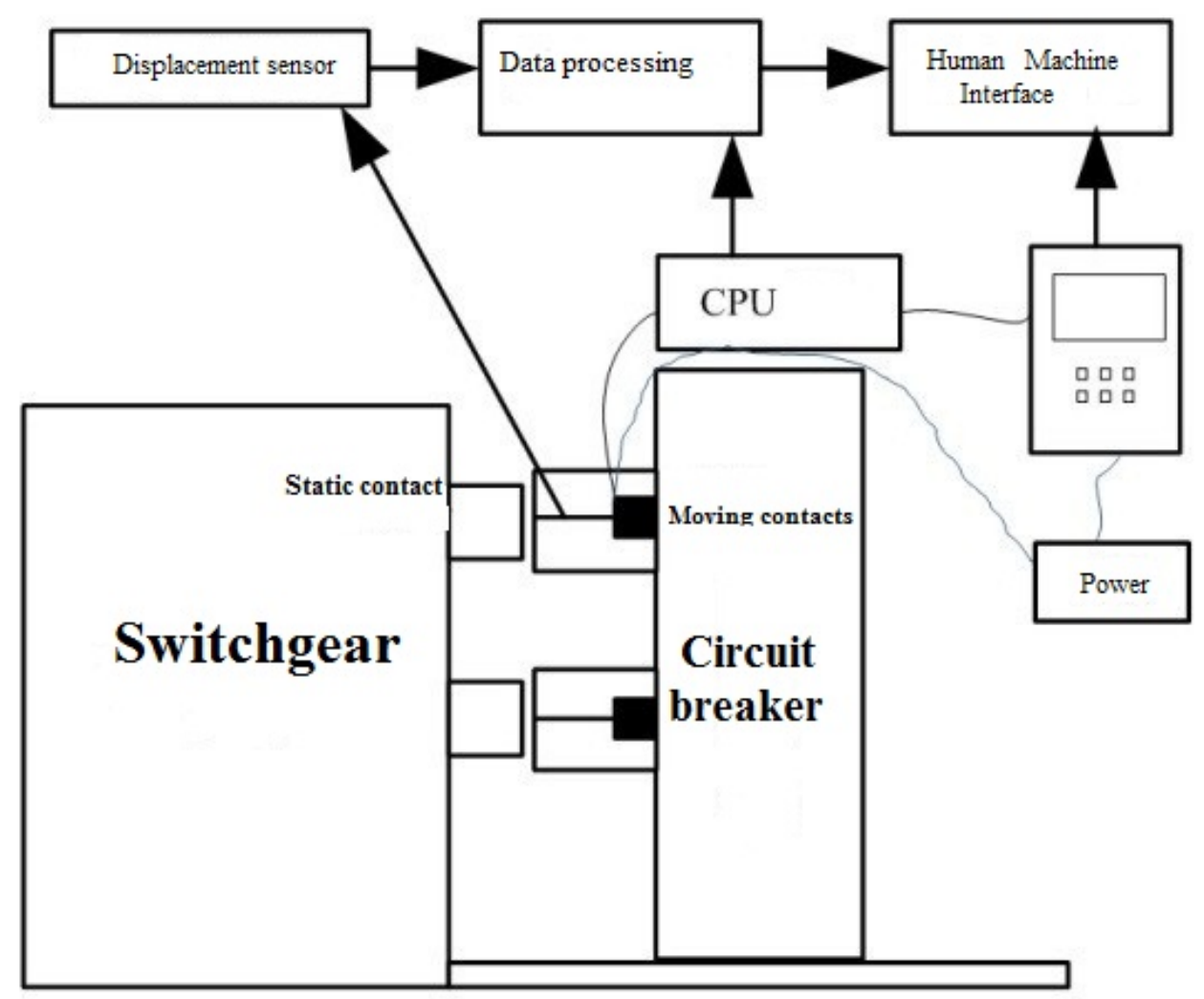

Fig.1 schematic diagram on depth of engagement intelligent measurement device

The depth of engagement intelligent measurement device for switchgear includes six sets of displacement sensor and mainframe box. Six sets of displacement sensors were installed in the six movable contacts internal counter bore of A, B, C three-phase of high voltage switchgear handcart circuit breaker, the power lines and signal lines were lead by a peripheral ring gap plum contacts and circuit breaker handcart, and connected to the data processing device, which connected to human machine interface display module. The displacement sensors includes contact type or guide rebound displacement sensors, sensor range is $0 \mathrm{~mm}-50 \mathrm{~mm}$, sensor accuracy is $0.1 \%-1 \%$, the sensor is $\mathrm{DC} 5 \mathrm{~V}$, the output voltage $0-5 \mathrm{~V}$.

\section{Component Selection}

Select bit-contact displacement sensor or guide rebound displacement sensor. Its structure diagram is shown in Figure 2. Sensor range is $0-50 \mathrm{~mm}$, and the accuracy is $\pm 0.1 \%$. Displacement sensor input and output interface is USB interface, the signal line extended using USB data line. DC power is $12 \mathrm{~V}$ adjustable voltage grade. DC power is equipped with a charger and charging outlet. Excitation signal of sensor is supplied by $5 \mathrm{~V}$ DC power voltage excitation. Displacement output signal of the sensor is voltage-form output, after data processing by mainframe box. The result is displayed on the displacement display instruments. Accuracy of measurement and display is \pm $0.1 \mathrm{~mm}$.

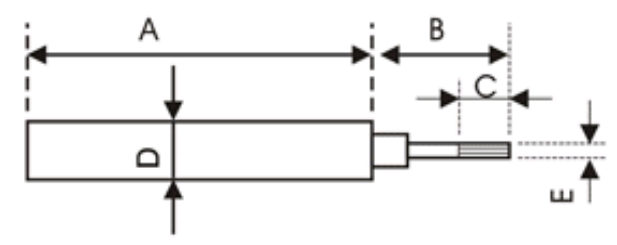

Fig.2 Schematic diagram of the sensor 


\section{Mainframe box trial}

The mainframe box is the key component of measurement device, an internal data input USB connector, the data processing apparatus, data display instrument, DC power, chargers and various auxiliary connecting means are installed in it.

The shell of mainframe box is made by aluminum alloy, the front and rear panel of mainframe box is customized. The front panel layout is shown in Figure 3. The input voltmeter, potential regulator and output signal (A1, B1, C1, A2, B2, C2) mounting holes are set. The rear panel layout is shown in Figure 4. The input signals USB interface (A1, B1, C1, A2, B2, C2), power supply switch and power socket holes are set. The overall size of the front and rear panels are 320mm long, 130mm high.

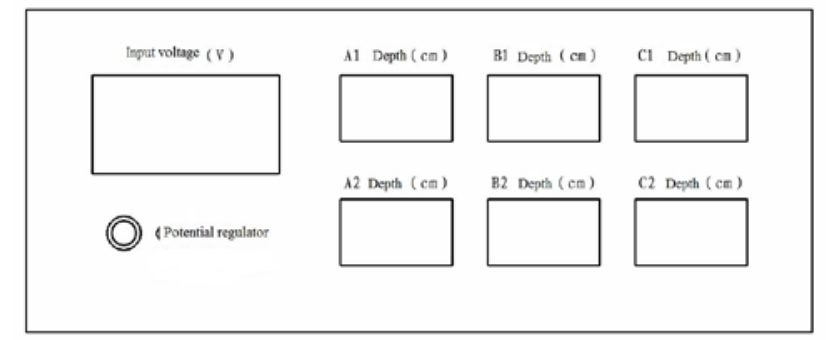

Fig.3 The front panel layout

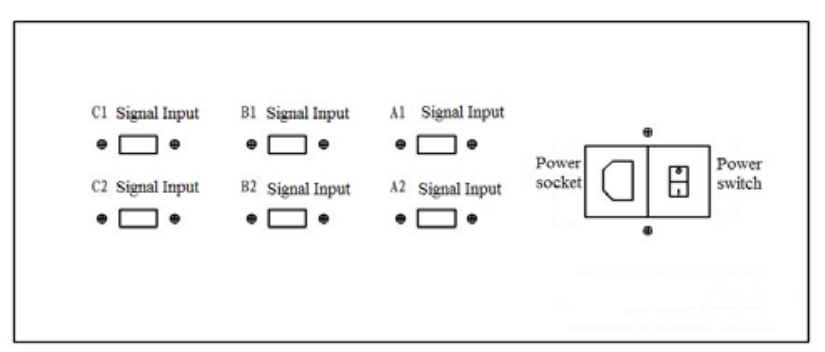

Fig.4 The rear panel layout

\section{Auxiliary workpiece trial}

In order to install and fix displacement sensor into the inner hole of handcart circuit breaker dynamic contacts, the displacement sensor assisted artifacts are designed and produced, including two-semi type fixtures and mandrel auxiliary devices. Two-semi type device can be easily fixed the sensor into the inner hole of handcart circuit breaker dynamic contacts; mandrel auxiliary device can ensure that the displacement of the displacement sensor and depth of engagement are synchronous.

\section{Test}

Figure 5 shows a photograph of bench test. Mainframe box of measuring device is in the upper part; the simulation test bench of handcart circuit breaker engagement process is in the lower part, which equipped with a set of static and dynamic contacts, and displacement sensor is inside the dynamic contacts. Specific simulation works as follows: When the circuit breaker handcart push switchgear, the dynamic contact go into the static contact, then start counting, that is, when the mandrel of displacement sensor touching static contact, start counting until the trolley moved to the working position. This section is called the depth of engagement of static and dynamic contacts, the stroke of the displacement is detected by the sensor and output, the data processing apparatus reading and processing, and ultimately the displacement data can be displayed on the output display instruments. The staff would determine whether depth of engagement of dynamic contacts of high voltage switchgear circuit breaker handcart meet requirements, if meet the requirements, the handcart can be removed, the measuring device can be dismantled, and reset the handcart working position; if not, require staff to remove the trolley, according to the need to increase or decrease the number of static contact mounting pads, and then repeat the work until it meets the requirement.

In addition, through the field trials, the trial results show that the measuring device is easy to operate, accurate measurement, metal-enclosed armored meet the high voltage switchgear circuit breaker plug handcart depth of engagement intelligent measurement requirements. Measuring range of the measuring device is $0-50 \mathrm{~mm}$, measurement and display accuracy is $\pm 0.1 \mathrm{~mm}$. 


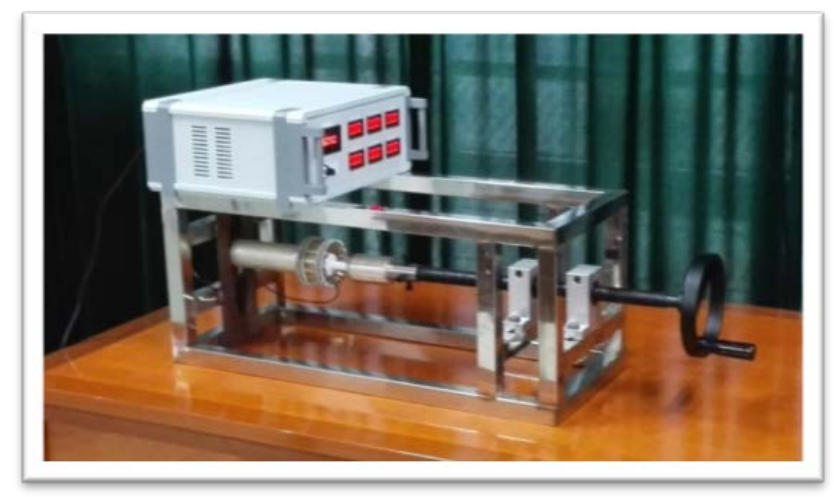

Fig.5 Bench test photo of intelligent measuring device

\section{Summary}

A novel depth of engagement intelligent measurement device for handcart circuit breaker contacts about metal-enclosed armored high voltage switchgear was developed. The device includes six sets of precision displacement measurement sensor and the main instrument chassis. The device structure is reasonable, easy to measure, accurate. Measuring range of the measuring device is $0-50 \mathrm{~mm}$, measurement and display accuracy is $\pm 0.1 \mathrm{~mm}$.

\section{Acknowledgment}

This work was financially supported by a project Funded by the Priority Academic Program Development of Jiangsu Higher Education Institutions (PAPD), Jiangsu Electric Power Company Science and Technology Project (No. 10KJ019901C207912013000) and the Nantong University Natural Science Foundation (No.14H061).

\section{References}

[1] D.X. Zhang, L.Z. Yao, W.Y. Ma. Development strategies of smart grid in China and abroad[J].Proceedings of the CSEE, 2013,33(31):1-14 ( in Chinese).

[2] M.J. Wang. Smart grid and smart energy resource grid[J].Power System Technology, 2010, 34(10):1-5( in Chinese).

[3] X.B. Feng. Discussion on intelligent substation [J]. High Voltage Apparatus,2013,49(2):116119( in Chinese).

[4] N.N. Song, N. Tong, X.J. Yan, et al. Discussion on Some Problems of Smart Grid[J]. Jiangsu Electrical Engineering, 2010(03): 82-84( in Chinese).

[5] J. Liu, D.F. Ju, Y. Wang, et al. Design and application of intelligent condition monitoring system for substation equipment[J]. Electric Power Construction, 2012,33(6):6-10( in Chinese).

[6] S. Wang, X.H. Wu, P. Yuan, et al. Current situation and development of calibration for UHF-PD(partial discharge) detection system[J]. High Voltage Apparatus, 2007,43(1): 59-64( in Chinese).

[7] J.Y. Dong, H. Yao, H.J. Liu, et al. Key characteristics and prospect of large-scale application of electric transmission and transformation equipment on-line monitoring[J]. Electrotechnics Electric, 2014(08):6-10( in Chinese).

[8] Y. Jin, W. Liu, X.G. Zha, et al. The technology of state monitoring system and its application in smart substation[J]. Jiangsu Electrical Engineering, 2012(02):12-15( in Chinese).

[9] Q. Liu, C. Cheng, J. Wu, et al. Design and implementation of temperature monitoring system for intelligent substation[J]. Power System Protection and Control, 2013, 41(4): 130-135( in Chinese). 